\title{
Mesoscale temperature fluctuations in the Southern Hemisphere stratosphere
}

\author{
B. L. Gary \\ Jet Propulsion Laboratory, Pasadena, CA, USA \\ 5320 E. Calle Manzana, Hereford, AZ 85615-9514, USA
}

Received: 17 March 2008 - Published in Atmos. Chem. Phys. Discuss.: 21 May 2008

Revised: 18 July 2008 - Accepted: 18 July 2008 - Published: 14 August 2008

\begin{abstract}
Isentrope surfaces in the Southern Hemisphere stratosphere reveal that air parcels undergo mesoscale temperature fluctuations that depend on latitude and season. The largest temperature fluctuations occur at high latitude winter, whereas the smallest fluctuations occur at high latitude summer. This is the same pattern found for the Northern Hemisphere stratosphere. However, the amplitude of the seasonal dependence in the Southern Hemisphere is only $37 \%$ of the Northern Hemisphere's seasonal amplitude.
\end{abstract}

\section{Introduction}

A companion article, "Mesoscale Temperature Fluctuations in the Stratosphere" (Gary, 2006), describes an analysis of stratospheric isentrope "wrinkles" in the Northern Hemisphere (NH) using measurements by the Microwave Temperature Profiler (MTP) aboard NASA ER-2 and DC-8 aircraft. The amplitude of mesoscale temperature fluctuations, referred to hereafter as "mesoscale fluctuation amplitude" (MFA), is defined as the full-width at half-maximum of a histogram of departures of an isentrope surface altitude from its synoptic scale counterpart. The companion article shows that in the NH stratosphere MFA depends on the following four independent variables: latitude, season, underlying topography and altitude. Figure 1, taken from that article, shows the latitude dependence of MFA for two seasons.

The present article is also based on measurements by the MTP. However, all flights in this analysis are in the Southern Hemisphere (SH). The purpose of the present analysis

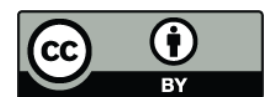

Correspondence to: B. L. Gary

(blgary@umich.edu) is to determine if MFA in the SH has the same dependencies on latitude and season that were found for the NH. The NH MFA analysis employed ER-2 and DC-8 measurements at altitude regimes centered on 19 and $11 \mathrm{~km}$, and therefore permitted an investigation to be made of the altitude dependence of MFA. All SH data were made using only an ER-2 so it is not possible in this study to evaluate the dependence of SH MFA on altitude. It was also not possible to evaluate the dependence of SH MFA on topography because all flights were over ocean.

The archive for this study consists of 22 ER-2 flights, most of which were based in Christchurch, New Zealand. Since most flights were north-south at a fixed longitude, and since MFA was suspected to depend on latitude, each flight was divided into segments with a latitude span of $\sim 10$ degrees. This usually meant that each flight was divided into four segments of 1.5-h duration, producing a total of 81 flight segments; 75 were over ocean and 6 were over New Zealand. The median altitude for all flight segments is $19.2 \mathrm{~km}$, with a range of 17.8 to $20.4 \mathrm{~km}$.

\section{Remote sensor}

The Microwave Temperature Profiler aboard the ER-2 aircraft is a passive radiometer operating at frequencies 56.66 and $58.80 \mathrm{GHz}$. At these frequencies atmospheric opacity is dominated by oxygen molecules. At $19 \mathrm{~km}$ altitude the opacities for the two frequencies are $\sim 0.4$ and 0.9 Nepers $/ \mathrm{km}$; the corresponding atmospheric source functions have exponential weighting functions with ranges of 2.5 and $1.1 \mathrm{~km}$, respectively. A 45-degree reflector is rotated through a sequence of 10 elevation angles, including the horizon. At each elevation setting brightness temperature is measured at both frequencies. After measurements of sky brightness

Published by Copernicus Publications on behalf of the European Geosciences Union. 


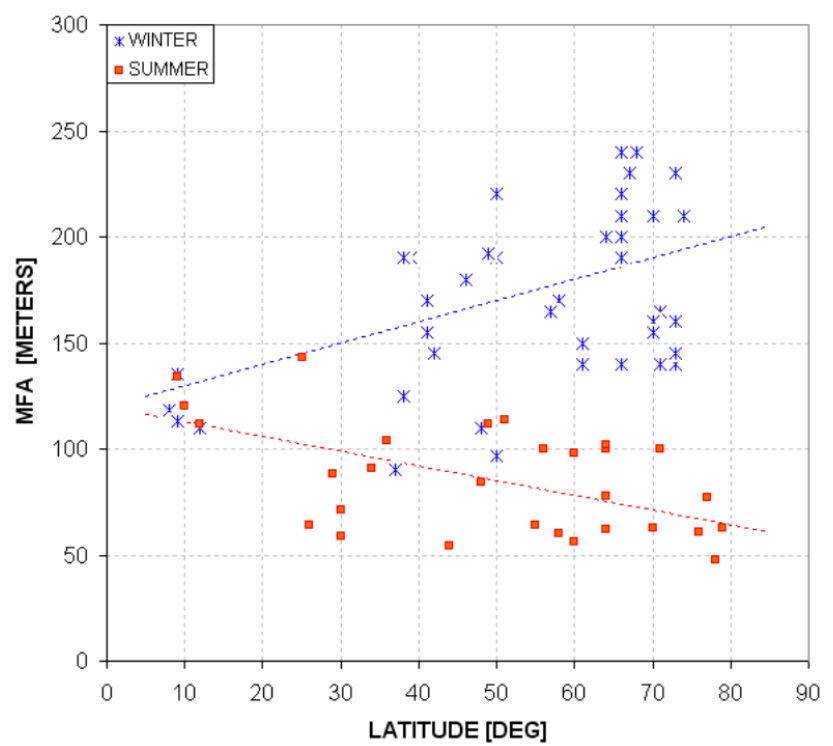

Fig. 1. Northern Hemisphere measurements of MFA versus latitude for winter (blue asterisks) and summer (red squares). This graph appears in the companion article.

temperature are made, the reflector is rotated for viewing of an ambient target. An observing cycle requires $\sim 20 \mathrm{~s}$ to complete; this means that the MTP produces an altitude temperature profile at intervals of $\sim 4 \mathrm{~km}$ along the flight path. Statistical retrieval coefficients are calculated prior to deployment and these are used to convert the 20 observed brightness temperatures per observing cycle to a temperature profile. The derived profile of temperature versus altitude extends from $\sim 3$ below flight level to $\sim 4 \mathrm{~km}$ above. Additional information about the remote sensing concepts employed by the MTP, as well as observing and retrieval analysis procedures, are presented in the companion paper.

\section{Data processing}

MTP measurements for both hemispheres were processed the same way. Individual $T(z)$ profiles are obtained along flight paths that extend $\sim 2700 \mathrm{~km}$ before mid-flight turn-around, corresponding to $\sim 26$ degrees of latitude for a north/south flight. The outbound and inbound flight segments are at different altitudes, and the MTP data for each is used to create an "isentrope altitude cross-section" (IAC).

It is possible to create a synoptic scale IAC for the same flight path, at the time of the flight, using a global data base of analyzed data from radiosondes and satellite temperature profiles. When this is done the two IACs are in approximate agreement except for two differences: 1) the MTP IAC shows much more spatial structure since it has a horizontal spatial resolution that is about 100 times better, $\sim 4 \mathrm{~km}$ versus $\sim 400 \mathrm{~km}$, and 2) there are altitude offsets between the two IACs that persist for hundreds of km, typically, before they change sign. Because the ER-2 payload includes a high quality outside air temperature (OAT) sensor it is possible to determine that the MTP IAC is invariably the correct one. Even the short timescale high spatial frequency IAC structure of the MTP near flight level is in agreement with the OAT sensor. For this reason it is preferable to smooth the MTP data instead of using the synoptic scale analyzed data for the purpose of determining a mesoscale IAC. To convert a high spatial resolution MTP IAC to a synoptic version the measured IAC is smoothed using a $400-\mathrm{km}$ boxcar. A second 400-km boxcar smoothing step is performed, which produces structures that resemble a Fourier decomposition and reconstitution using only the $400 \mathrm{~km}$ and longer components. Figure 2 illustrates a typical relationship between MTP-measured isentropes and their $400 \mathrm{~km}$ double boxcar synoptic scale representation.

By subtracting the MTP-measured IAC from its counterpart synoptic scale version (the $400-\mathrm{km}$ double boxcar smoother version), it is possible to produce a "mesoscale only" IAC. This IAC is free of the systematic errors that would exist if a synoptic scale IAC were used that had been produced from the satellite and radiosonde-based analyzed data. It is a simple procedure to characterize the fluctuations of any flight segment of "mesoscale only" isentrope altitude for a specific isentrope by converting the values to a histogram and measuring the histogram's full-width at half-maximum, FHWM. This was done using a spreadsheet, where fitting the histogram with a Gaussian function was done manually. In almost every case the Gaussian function is a good fit to the observed histogram. Figure 3 is a typical histogram plot that illustrates these differences and the proper fit of Gaussian representations used to estimate MFA.

\section{Model for Mesoscale Fluctuation Amplitude depen- dencies}

MFA was determined from Gaussian fits to mesoscale minus synoptic scale isentrope altitudes for 81 flight segments of 22 ER-2 flights in the Southern Hemisphere. Each MFA value was entered into a spreadsheet with latitude and date. A season parameter called "Wintriness" was calculated for each entry that varied in a sinusoidal manner from 0.0 on 22 July to 1.0 on 22 January (described in detail in the companion article). An MFA was categorized as belonging to "summer" if Wintriness $<0.4$, and it was categorized as "winter" when Wintriness $>0.6$. Wintriness values between 0.4 and 0.6 were categorized as "spring".

Figure 4 shows the individual MFA measurements plotted versus latitude, with different symbols for winter and summer seasons. This figure exhibits a much greater seasonal overlap of individual MFA measurements for all latitude regions compared with measurements for NH (Fig. 1). To aid in illustrating the presence of a seasonal effect the 


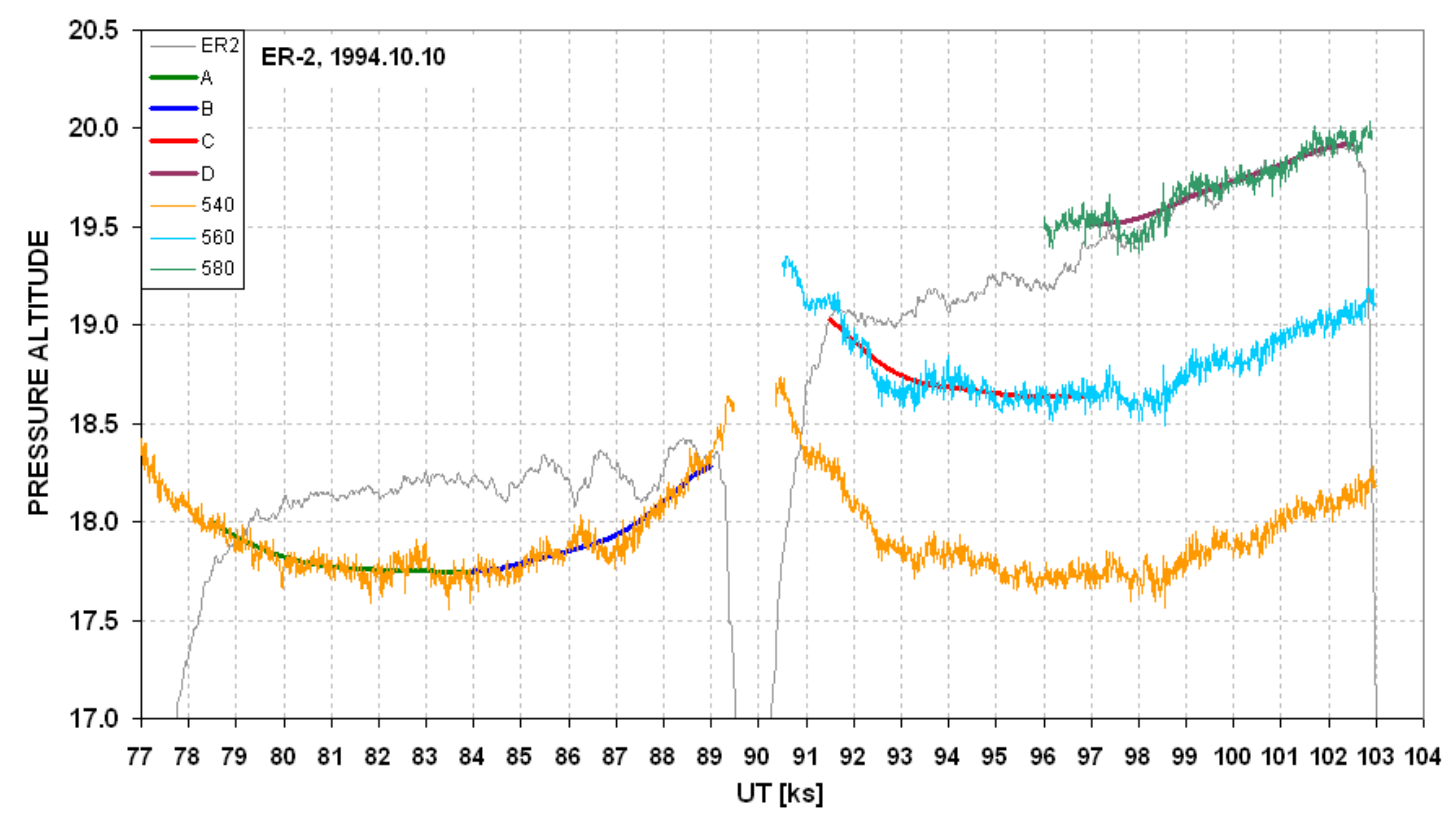

Fig. 2. Isentrope altitude cross-section for an ER-2 flight based in Christchurch, NZ. Altitudes for 540, 560 and $580 \mathrm{~K}$ isentrope surfaces are also represented by synoptic scale versions (smooth thick traces, flight segments A, B, C and D) using a $400 \mathrm{~km}$ double boxcar averaging procedure. The ER-2 flight altitude (light gray trace) shows a brief descent to $\sim 15 \mathrm{~km}$ (made for in situ air sampling) at the south end of the flight (at $\sim 90$ kiloseconds).
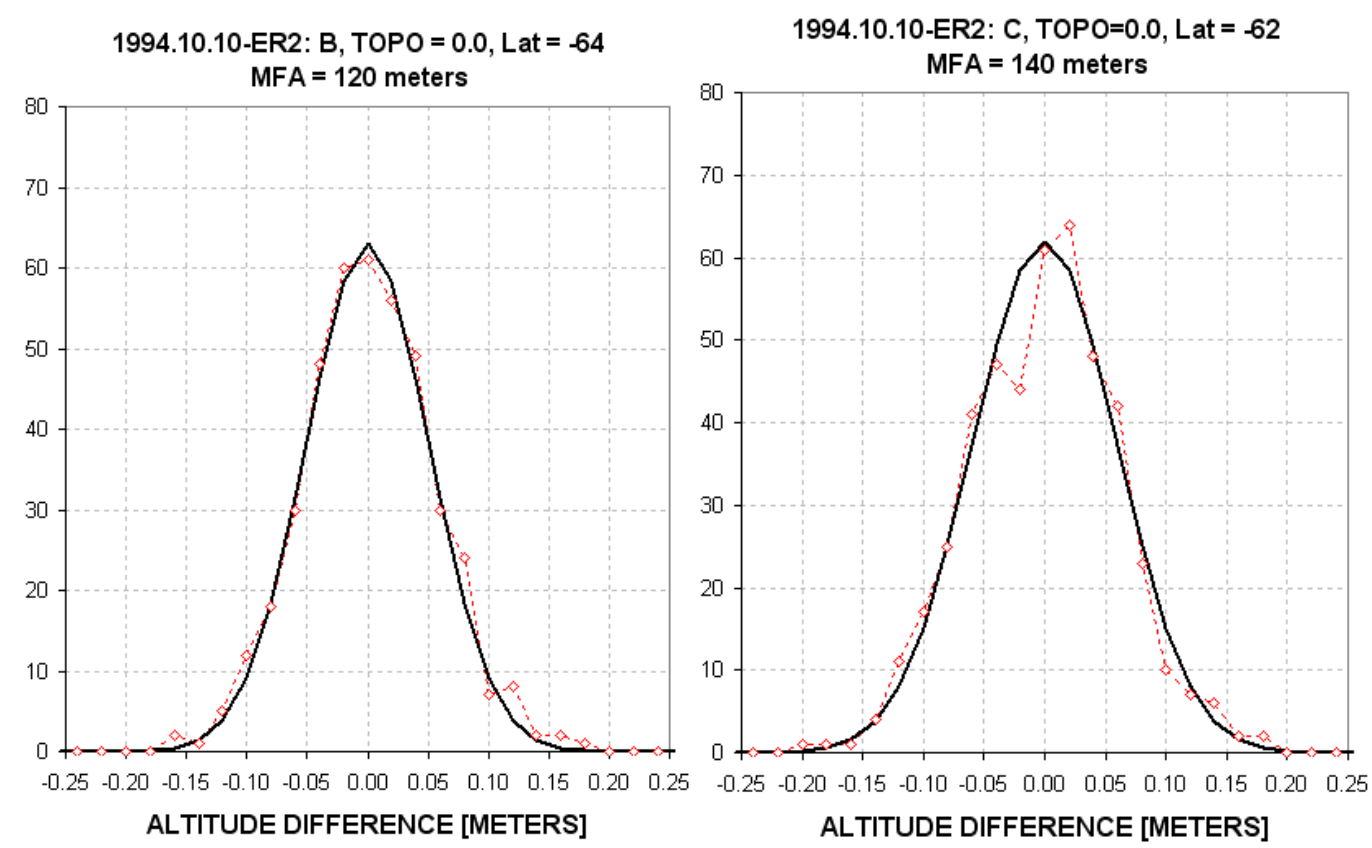

Fig. 3. Histograms of differences between mesoscale isentrope altitudes and synoptic scale altitudes for flight segments B and C for the ER-2 flight of 10 October 1994.

MFA entries for a season were arranged by latitude in groups of $\sim 6$ and a median combine of MFA was calculated. The median combine MFA values show a clear pattern of greater MFA in winter compared with summer. There is also weak evidence for an increasing seasonal difference with latitude. Both correlations are present in the NH MFA measurements (Fig. 1). 


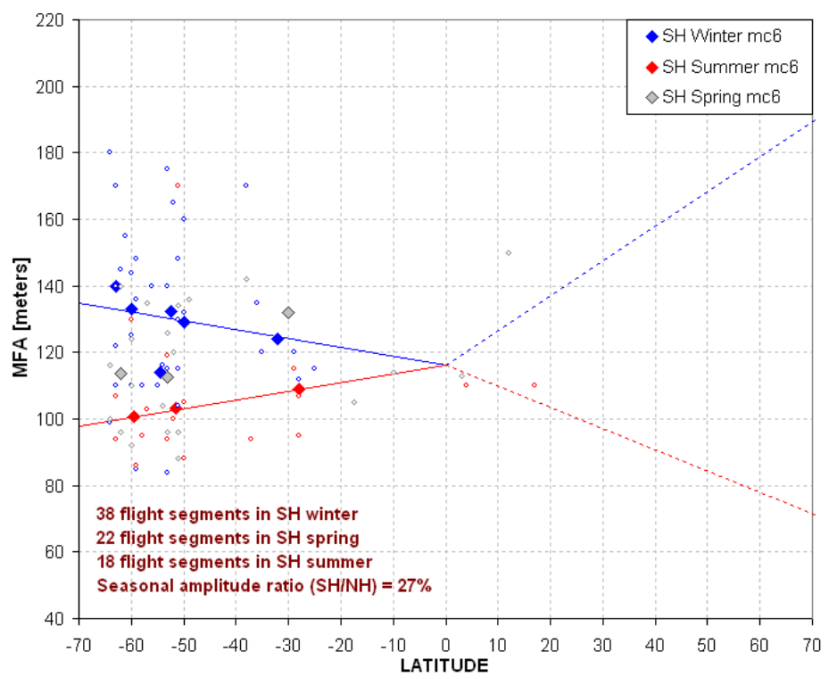

Fig. 4. Southern Hemisphere measurements of MFA during winter (blue), spring (gray) and summer (red). The large solid-color diamonds are a median combine of 6 individual MFA measurements (grouped by latitude). The dashed lines for $\mathrm{NH}$ latitudes correspond to the model fits to $\mathrm{NH}$ measurements as described in the companion article. All MFA have been adjusted to a $19.4 \mathrm{~km}$ reference altitude using an altitude exponent of -0.40 (discussed in the text).

The straight line fits to the summer and winter MFA values in the SH data (Fig. 4) are described by the following equations:

MFA_winter $[$ meters $]=116-0.27 \times$ Latitude $[\mathrm{deg}]$

MFA_summer [meters] $=116+0.26 \times$ Latitude $[\mathrm{deg}]$

The dependence on latitude of SH summer and SH winter MFA are approximately equal but of opposite sign. The same relation was found for $\mathrm{NH}$ slopes. The major difference between the SH and NH measurements is that the latitude dependence for the $\mathrm{SH}$ is $27 \pm 8 \%$ of the $\mathrm{NH}$ latitude dependence.

A multiple regression model was created that incorporates latitude and the seasonal parameter as independent variables. The dependent variable was MFA corrected for altitude. Since the range of altitudes was small an altitude correction exponent could not be solved for, so the correction equation found from $\mathrm{NH}$ data was used for correcting the $\mathrm{SH}$ MFA:

MFA_corrected $=\mathrm{MFA} \times(P[\mathrm{hPa}] / 58.85)^{-0.40}$

where $P$ is air pressure at the isentrope's altitude.

The range of altitude corrections was $17 \%$ (maximum minus minimum correction). The uncertainty of this correction is considered small compared with the correction, and it is especially small compared with the uncertainty of individual MFA measurements.

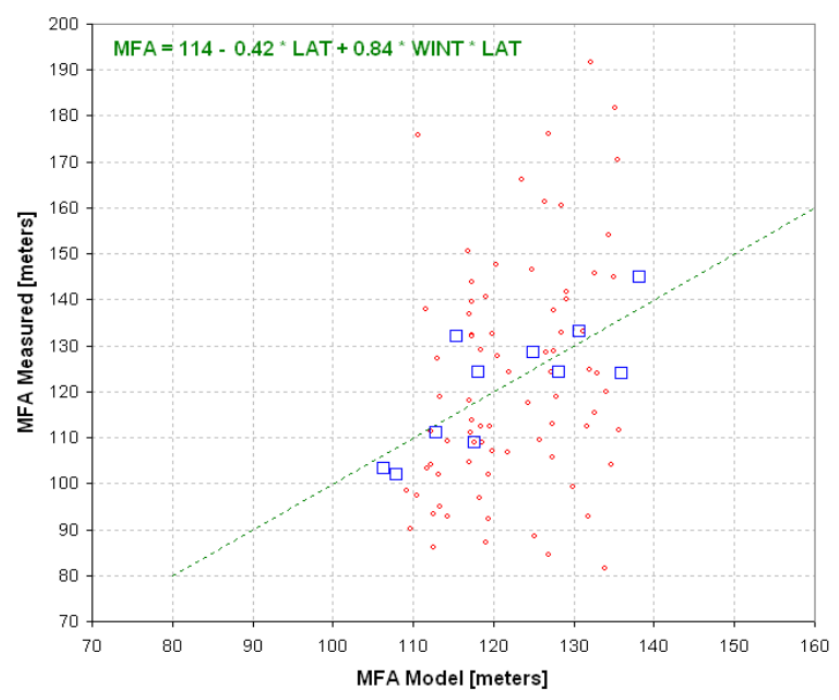

Fig. 5. Measured MFA (for SH) versus MFA predicted by model that includes a dependence on latitude, season and altitude. The blue squares are median combined versions of 7 individual flight segment measurements (using predicted MFA for grouping). Rsquared $=0.64$ for the median combined values.

The plot of measured versus model MFA in the SH is shown in Fig. 5.

The model line in this figure is described by the following equation:

$$
\begin{aligned}
\text { SH MFA [meters }] & =114-0.42 \times \text { Latitude }[\mathrm{deg}] \\
& \pm 0.17 \\
& +0.84 \times \text { Wintriness } \times \text { Latitude }[\mathrm{deg}] \\
& \pm 0.22
\end{aligned}
$$

The companion article concluded with a similar equation except that the coefficients are different:

$$
\begin{aligned}
\text { NH MFA [meters] } & =112-1.21 \times \text { Latitude }[\mathrm{deg}] \\
& \pm 0.27 \\
& +2.20 \times \text { Wintriness } \times \text { Latitude }[\mathrm{deg}] \\
& \pm 0.20
\end{aligned}
$$

This version of the NH model corresponds to flight over ocean ("topography parameter" = zero) and at ER-2 altitudes $(19.4 \mathrm{~km})$. It is apparent that the SH coefficients are significantly smaller than the corresponding $\mathrm{NH}$ coefficients. The ratio of the latitude coefficients is $0.35 \pm 0.15$. The ratio of the "Wintriness $\times$ Latitude" terms is $0.38 \pm 0.09$. It is significant that the signs of corresponding terms are the same, and the constant terms (114 and 112) are essentially the same.

\section{Conclusion}

"Mesoscale temperature fluctuations" can be inferred from the amplitude of mesoscale "wrinkles" of isentrope altitude cross-sections. The mesoscale fluctuation amplitudes (MFA) 
of these wrinkles is smaller in the Southern Hemisphere than the Northern Hemisphere. When allowance is made for the dependence of MFA upon latitude, season, topography and altitude, it is found that the ratio of SH to NH MFA is $37 \pm 11 \%$.

Acknowledgements. The research described in this publication was carried out by the Jet Propulsion Laboratory, California Institute of Technology, under a contract with the National Aeronautics and Space Administration. Specific acknowledgement is made for the contributions of R. Denning for his instrument construction and support throughout all field uses of the Microwave Temperature Profiler. I thank M. J. Mahoney for assuming all MTP responsibilities after my retirement and for his encouragement to bring this work to publication.

Edited by: B. Kärcher

\section{References}

Gary, B. L.: Mesoscale temperature fluctuations in the stratosphere, Atmos. Chem. Phys., 6, 4577-4589, 2006,

http://www.atmos-chem-phys.net/6/4577/2006/. 